\title{
CARACTERÍSTICAS INFLAMATORIAS DE LA OBESIDAD
}

\section{INFLAMMATORY CHARACTERISTICS OF OBESITY}

\author{
Marcela Reyes J. \\ Unidad Académica de Salud Pública, Nutrición y Epidemiología Genética. \\ Instituto de Nutrición y Tecnología de los Alimentos (INTA), Universidad de Chile. Santiago, Chile.
}

\begin{abstract}
Currently, obesity is considered a low chronic inflammatory state. The alterations that occur into the expanding adipose tissue parallel the ones seen in a classic inflammatory process. However, the stimulus that triggers inflammation is still unknown. The consequences of the immune system activation are poorly understood. The inflammatory process is not just present at the adipose tissue level. In fact, it can be found in circulation, pancreas, liver and muscle (among other organs). Moreover, inflammation is playing a role on the pathophysiology of most of cardiovascular risk factors linked to obesity. The aim of this article was to review the current knowledge about this important topic. The understanding of the immune process linked to obesity would allow its clinic study and even the intervention. Key words: adipose tissue; cardiovascular risk; immune system; inflammation.
\end{abstract}

Este trabajo fue recibido el 28 de Diciembre de 2009 y aceptado para ser publicado el 15 de Noviembre de 2010.

\section{INTRODUCCIÓN}

Un balance energético positivo que se mantiene en el tiempo se traduce en la expansión del tejido adiposo, característica fundamental de la obesidad. Aunque está ampliamente descrito que la obesidad condiciona morbimortalidad cardiovascular (1), aún no existe claridad sobre la asociación fisiopatológica que existe entre el aumento en el volumen del tejido adiposo y el desarrollo de los factores de riesgo cardiovascular y del proceso de ateromatosis. En relación a esto, se piensa que las características biológicas que tenga la expansión de la masa grasa determinarán su potencial patogénico. El tejido adiposo entre los sujetos obesos puede diferir en muchas de sus propiedades, como son la ubicación, el desarrollo de la red vascular, el perfil de síntesis y secreción de adipoquinas, la actividad lipolítica y lipogénica, el potencial de adipogénesis (reclutamiento de células preadiposas para su paso a adipocitos maduros), la infiltración por células inmunes, entre otras. Estas características están íntimamente relacionadas entre sí, potenciándose entre ellas, pudiendo generar círculos viciosos que condicionen un tejido adiposo patológico. Es así como el tejido adiposo visceral tendría un peor perfil secretor de adipoquinas, determinando una mayor atracción de células inflamatorias, las que aumentan la lipólisis y disminuyen la adipogénesis; al estar desfavorecida la maduración de nuevas células adiposas, aumentan los adipocitos hipertróficos que presentan un peor perfil secretor. En contraposición, podrían establecerse círculos virtuosos de expansión tisular, determinando un tejido adiposo más benigno y, por lo tanto un sujeto obeso, pero metabólicamente sano.

\section{ALTERACIONES INFLAMATORIAS DEL TEJIDO ADIPOSO}

El tejido adiposo de los obesos posee un número aumentado de células inflamatorias, lo que se observa tanto en modelos animales como humanos de obesidad (2). Se desconoce aún el estímulo primario que gatilla el fenómeno inflamatorio del tejido adiposo, aunque la evidencia actual nos permite plantear que sus características son muy similares a cualquier otro proceso inflamatorio agudo que se hace crónico, involucrando modificaciones del endotelio local que permiten el paso selectivo de neutrófilos, macrófagos y linfocitos.

No existe claridad sobre el curso temporal de los 
fenómenos inflamatorios, así como sobre el papel que juega la presencia de cada uno de los distintos leucocitos en la fisiopatología de la obesidad. Sin embargo se ha determinado que la acumulación de macrófagos en el tejido adiposo tiene consecuencias nefastas en el metabolismo local y sistémico. En efecto, en los modelos de obesidad murina en los que se evita la infiltración por macrófagos en el tejido adiposo a través de la ablación génica de diferentes moléculas pro-inflamatorias, los animales no desarrollan las alteraciones metabólicas sistémicas propias de la obesidad $(3,4)$. Por otro lado, animales normopeso en los que se aumenta el contenido de macrófagos de este tejido, sí desarrollan estas alteraciones (5). No solo las manipulaciones cuantitativas de los macrófagos tisulares repercuten en el metabolismo sistémico, también lo hacen las manipulaciones en el estado inflamatorio de estas células, aunque su número no cambie. De esta forma, la ablación de proteínas proinflamatorias de forma selectiva en las células mieloides (precursores de monocitos/macrófagos) inducen mejoría metabólica sistémica en ratones obesos (6), mientras que cuando se tornan más inflamatorias (al evitar la expresión de mediadores antiinflamatorios por parte de las células) el metabolismo sistémico empeora (7). Antecedentes recientes permiten plantear un escenario similar en obesidad humana: un mayor contenido de macrófagos en el tejido adiposo de sujetos obesos fue el principal determinante de las alteraciones en la sensi- bilidad insulínica cuando se compararon sujetos obesos con o sin insulino-resistencia (8). Las consecuencias metabólicas de la presencia aumentada de linfocitos en el tejido adiposo son menos claras, siendo la evidencia aún incipiente y no del todo concluyente. Estas células inmunes tendrían un papel modulador de la quimiotaxis y función de los monocitos/macrófagos. Los linfocitos T citotóxicos (DC8+) exacerbarían la inflamación (empeorando el metabolismo) $(9,10)$, mientras que los del subtipo ayudador o helper (CD4+) la disminuirían (10). Asimismo, existe poca información sobre el papel que los neutrófilos podrían jugar en la inflamación del tejido adiposo; al parecer su presencia es solo transitoria (11).

\section{CONSECUENCIAS LOCALES DE LA INFLAMACIÓN DEL TEJIDO ADIPOSO}

A nivel local, la acumulación de macrófagos tiene efectos sobre la fisiología de los adipocitos y las células preadiposas, generando una comunicación intercelular que exacerba el funcionamiento patológico de este tejido (figura 1). Los factores derivados de macrófagos (en particular el factor de necrosis tumoral alfa, TNF $\alpha$ ) modifican el perfil de expresión y secreción de adipoquinas por parte de las células adiposas (12), tornándolo en uno que condiciona insulino-resistencia, aterogénesis e inflamación. Los productos secretados por el tejido adiposo no solo tienen consecuencias autocrinas, sino que también tienen repercusiones sistémicas (acción en-

\section{FIGURA 1}

Efecto de la presencia aumentada de macrófagosen la fisiología de las células adiposas.

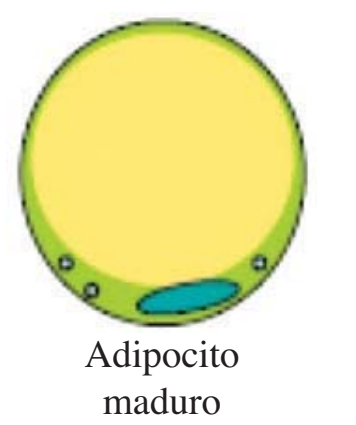

- Aumento secreción adipoquinas pro-inflamatorias y pro-trombóticas

- Resistencia insulínica

- Lipólisis

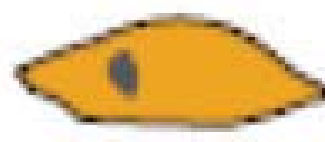

Preadipocito
- Aumento secreción adipoquinas proinflamatorias y protrombóticas

- Resistencia insulínica

- Disminución adipogénesis 
docrina) y en los órganos adyancentes (acción paracrina). Además del cambio inducido en las células adiposas, los productos derivados de macrófagos actúan en el metabolismo local de lípidos y glucosa, al inducir lipólisis (13) e insulino-resistencia (14). Por otra parte, la presencia de macrófagos empeora la adipogénesis, transformándose en un estímulo inhibitorio de la diferenciación de preadipocitos a adipocitos maduros (15). Se piensa que cuando está limitada la expansión producto de la hiperplasia (generación de nuevas células adiposas maduras) el tejido adiposo solo puede expandirse producto de la hipertrofia celular (16); si el balance energético positivo se mantiene, el tejido adiposo puede hacerse relativamente incompetente en el almacenaje de triglicéridos, depositándose estos ectópicamente (en la periferia de órganos o infiltrándolos) lo que conlleva a lipotoxicidad $\mathrm{y}$ al efecto nocivo deletéreo paracrino de las adipoquinas antes señalado. Por lo tanto, los macrófagos aumentados en el tejido adiposo inician y/o exacerban las alteraciones tisulares locales propias de la obesidad.

\section{EVIDENCIA DE INFLAMACIÓN FUERA DEL TEJIDO ADIPOSO}

La obesidad también condiciona un estado inflamatorio a nivel sistémico. Éste se manifiesta al medir los mediadores inflamatorios en circulación como son las proteínas de fase aguda interleuquina (IL) 6 y la proteína $\mathrm{C}$ reactiva (PCR), además del TNFa y otras interleuquinas. Los niveles plasmáticos de los mediadores inflamatorios están asociados positivamente con la magnitud de los depósitos adiposos (índice de masa corporal, porcentaje de grasa corporal, circunferencia cintura) así como con las consecuencias metabólicas de la obesidad (insulino-resistencia, dislipidemia, presión arterial), tanto en población pediátrica como adulta $(17,18)$. No hay claridad si este aumento de los productos inflamatorios circulantes es consecuencia de la modificación inflamatoria del tejido adiposo y por lo tanto producidos por éste o, en el sentido opuesto, están jugando un papel en el aumento del número de leucocitos en circulación (al estimular la salida desde la médula ósea) o en la activación de estos leucocitos circulantes, favoreciendo así la infiltración del tejido graso.

Se ha postulado que los leucocitos circulantes estarían aumentados tanto en número como en el estado inflamatorio en los sujetos obesos. El recuento total de leucocitos está asociado a la adiposidad (19), de la misma forma que lo está el recuento diferencial de neutrófilos (20). Las diferencias numéricas son menos claras para los linfocitos y los monocitos. Las alteraciones cuantitativas de los leucocitos en obesidad son de escasa magnitud, necesitándose grandes tamaños muestrales para identifi- carlas; sin embargo, las modificaciones cualitativas son mucho más marcadas. Los leucocitos provenientes de sujetos obesos están en un estado pro-inflamatorio que se refleja en la activación del factor de transcripción inflamatorio NF-kB (21) y en la expresión de marcadores de superficie $(22,20)$. Esto se ha visto tanto en células mononucleares totales (que corresponden a los linfocitos y monocitos), como en monocitos aislados y en neutrófilos. Se ha descrito que la activación de estas células inflamatorias estaría dada por la ingesta de alimentos (23). Incluso, se sugiere que las características nutricionales de los alimentos ingeridos tendrían repercusiones en la magnitud de la alteración inflamatoria: dietas de mayor índice glicémico o con más ácidos grasos saturados tienen consecuencias inflamatorias de mayor relevancia (24). De esta manera, existe plausibilidad biológica para plantear tanto una activación sistémica de la inmunidad con posterior infiltración al tejido adiposo como un fenómeno local del tejido adiposo en expansión que luego se generaliza.

\section{CONSECUENCIAS DE LA ACTIVACIÓN SISTÉMICA DE LA INMUNIDAD}

Estudios en modelos murinos de obesidad sugieren que la activación del sistema inmune a nivel sistémico podría tener repercusiones en la génesis de patología asociada a obesidad. El aumento temprano en el número y estado inflamatorio de los monocitos circulantes en ratones sometidos a dieta grasa muestra asociación con las alteraciones inflamatorias del tejido adiposo y predice los niveles de insulino-resistencia presente en etapas tardías de la obesidad (25). De manera análoga, la magnitud de la monocitosis inducida por una dieta aterogénica se asocia al tamaño de la lesión ateromatosa en ratones (26). De hecho, se sabe que la inmunidad juega un papel fundamental tanto en la génesis como en la progresión del proceso ateromatoso (27).

Los datos en humanos son aún incipientes y no permiten determinar un curso temporal, como en los modelos animales. Al comparar población obesa con o sin factores de riesgo cardiovascular, vemos que los leucocitos del grupo con peor perfil metabólico tienen diferencias en las características cuanti y cualitativas $(22,28,29)$, sugiriendo un posible papel de la activación del sistema inmune en las consecuencias que tiene la obesidad sobre el metabolismo sistémico. Incluso se ha sindicado a los marcadores inflamatorios como predictores de morbimortalidad cardiovascular (19).

Aunque escapa a los propósitos de esta revisión, es pertinente mencionar que prácticamente todas las patologías metabólicas asociadas a la malnutrición por exceso tienen un componente inflamatorio dentro de su génesis 
y/o progresión. La activación de la vía inflamatoria en cualquier tipo celular (a nivel del factor de tanscripción NF-kB) entorpece la señalización celular de la insulina. La resistencia insulínica es la condición que subyace a todas las entidades del síndrome metabólico, pero además para cada uno de los factores de riesgo cardiovascular, existe una asociación fisiopatológica directa con la inflamación, como es el caso de diabetes (30), dislipidemia (31), disfunción endotelial e hipertensión (32) y esteatosis hepática no alcohólica (33) (figura 2).

\section{INTEGRACIÓN DEL METABOLISMO Y SISTEMA INMUNE}

La evidencia actual nos permite plantear que metabolismo e inmunidad están íntimamente ligados (34). De esta manera, estímulos metabólicos permiten activar el sistema inmune, el que a su vez presenta repercusiones en la homeostasis metabólica. Esta interconexión ha sido ampliamente investigada en los casos de déficit nutricional (35), en los que el sistema inmune muestra una función deteriorada (siendo las infecciones la principal causa de muerte en desnutrición). La evidencia actual sugiere que este fenómeno también ocurriría en la malnutrición por exceso. La vinculación entre ambos sistemas está dada, muy probablemente, por su origen evolutivo común: en seres evolutivamente inferiores (como drosófilas) el cuerpo graso es el órgano equivalente a lo que en mamíferos corresponde al tejido adiposo, hígado y sistema inmune. Este nexo filogenético determina que ambos sistemas tengan vías aferentes y eferentes comunes. De esta manera, células inmunes como los macrófagos pueden ser estimuladas por componentes nutricionales como ácidos grasos libres. De manera análoga, células con función metabólica como los adipocitos y los hepatocitos son estimulados por moléculas inflamatorias como el TNFa. La íntima coordinación entre ambos sistemas es la que permite una derivación de nutrientes al sistema inmune en condiciones de infección importante. Incluso a nivel intracelular, ambos procesos están asociados, es así como el metabolismo celular basado en la

\section{FIGURA 2}

Consecuencias sistémicas del estado inflamatorio asociado a obesidad.

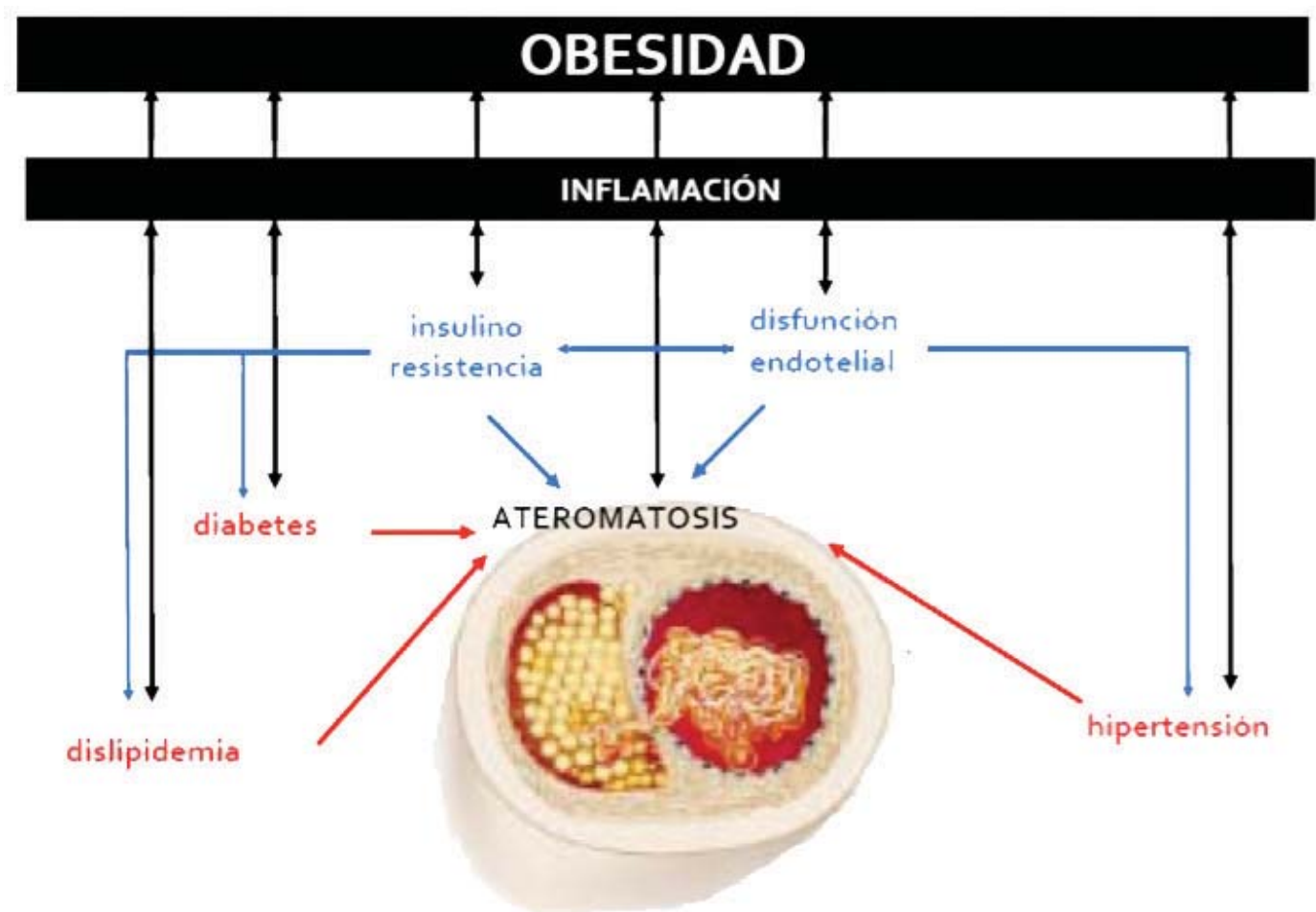

La inflamación es un actor relevante en la génesis de ateromatosis, tanto por su acción directa a nivel vascular como por su acción indirecta, condicionando resistencia insulínica y disfunción endotelial, así como diabetes, dislipidemia e hipertensión. 
oxidación de hidratos de carbono condiciona macrófagos inflamatorios, mientras que la utilización predominante de lípidos como sustrato energético condiciona en estas células inmunes un estado alternativo de activación, es decir, una célula con función anti-inflamatoria (36).

\section{IMPLICANCIAS CLÍNICAS}

Como se ha visto, la activación del sistema inmune parece ser trascendente en el desarrollo de factores de riesgo cardiovascular y enfermedad ateromatosa propiamente tal. Es por esto que las intervenciones que se pudieran hacer con la finalidad de impedir o mitigar la respuesta inflamatoria tendrían un importante efecto en la morbilidad asociada a obesidad. La manipulación de los eventos inflamatorios podría, al menos teóricamente, aumentar el porcentaje de obesos metabólicamente sanos, estimado en $30 \%$ (37). Sin embargo, el manejo deseable de la obesidad sigue siendo la baja de peso, la que tendría un efecto benéfico no tan solo en las alteraciones metabólicas y cardiovasculares, sino que también en las complicaciones de la obesidad asociadas a alteraciones osteoarticulares, neoplásicas, psicológicas, entre otras. La caracterización de la activación inflamatoria a nivel sistémico podría establecerse como un biomarcador que permita anticipar la evolución de los diferentes sujetos, a modo de anticipar las consecuencias de una ganancia activa de peso (25) o monitorizando los resultados de tratamientos dietarios (38).

\section{CONCLUSIONES}

La obesidad se asocia a un proceso inflamatorio crónico que afecta al tejido adiposo en sus diferentes localizaciones, pero que además es identificable a nivel circulatorio y en otros órganos como son hígado, páncreas, endotelio, entre otros. Su presencia y magnitud se asocia al desarrollo de factores de riesgo cardiovascular. La comprensión y caracterización de este fenómeno es de vital relevancia puesto que podría dar paso a intervenciones futuras que determinen una evolución favorable, en términos metabólicos, de los sujetos obesos.

\section{RESUMEN}

Actualmente la obesidad es considerada una enfermedad inflamatoria crónica, de intensidad leve. Las alteraciones que ocurren en el tejido adiposo en expansión son muy similares a las de una inflamación clásica, sin embargo se desconoce aún el agente lesivo que lo gatilla. La activación del sistema inmune no se da solamente en el tejido adiposo, sino que es identificable a nivel circulatorio y en los diferentes órganos relacionados con el metabolismo de sustratos como son hígado, páncreas y músculo. Los antecedentes actuales permiten plantear que tanto la génesis como la evolución de las enfermedades crónicas asociadas a la obesidad tienen un componente inflamatorio. El objetivo de este ensayo es revisar el conocimiento actual sobre el tema. Una cabal comprensión de la activación del sistema inmune en condiciones de exceso nutricional posibilitará su monitorización y eventual intervención.

Palabras clave: Obesidad; tejido adiposo; riesgo cardiovascular; sistema inmune; inflamación.

Dirigir la correspondencia a:

\author{
Profesora \\ Marcela Reyes J. \\ INTA - U. de Chile \\ Av. El Líbano 5524, Santiago, Chile. \\ Teléfono: 02-9781503 \\ Fax: 2214030 \\ E-mail: mreyes@inta.cl
}

\section{BIBLIOGRAFÍA}

1. Prospective Studies Collaboration, Whitlock G, Lewington S, Sherliker P, Clarke R, Emberson J, Halsey J, Qizilbash N, Collins R, Peto R. Bodymass index and cause-specific mortality in 900000 adults: collaborative analyses of 57 prospective studies. Lancet 2009; 373(9669):1083-96.

2. Weisberg SP, McCann D, Desai M, Rosenbaum M, Leibel RL, Ferrante AW Jr. Obesity is associated with macrophage accumulation in adipose tissue. $\mathrm{J}$ Clin Invest. 2003; 112(12):1796-808.

3. Weisberg SP, Hunter D, Huber R, Lemieux J, Slaymaker S, Vaddi K, Charo I, Leibel RL, Ferrante AW Jr. CCR2 modulates inflammatory and metabolic effects of high-fat feeding. J Clin Invest. 2006; 116:115-24.

4. Kim DH, Sandoval D, Reed JA, Matter EK, Tolod EG, Woods SC, Seeley RJ. The role of GM-CSF in adipose tissue inflammation. Am J Physiol Endocrinol Metab. 2008; 295(5):E1038-46.

5. Kamei N, Tobe K, Suzuki R, Ohsugi M, Watanabe T, Kubota N, Ohtsuka-Kowatari N, Kumagai K, Sakamoto K, Kobayashi M, Yamauchi T, Ueki K, Oishi Y, Nishimura S, Manabe I, Hashimoto H, Ohnishi Y, Ogata H, Tokuyama K, Tsunoda M, Ide T, Murakami K, Nagai R, Kadowaki T. Overexpression of monocyte chemoattractant protein-1 in adipose tissues causes macrophage recruitment and insulin resistance. J Biol Chem. 2006; 281(36): 26602-14.

6. Arkan MC, Hevener AL, Greten FR, Maeda S, Li ZW, Long JM, Wynshaw-Boris A, Poli G, Olefsky J, Karin M. IKK-beta links inflammation to 
obesity-induced insulin resistance. Nat Med. 2005; 11(2):191-8.

7. Odegaard JI, Ricardo-Gonzalez RR, Goforth MH, Morel CR, Subramanian V, Mukundan L, Red Eagle A, Vats D, Brombacher F, Ferrante AW, Chawla A. Macrophage-specific PPARgamma controls alternative activation and improves insulin resistance. Nature 2007; 447(7148):1116-20.

8. Klöting N, Fasshauer M, Dietrich A, Kovacs P, Schön MR, Kern M, Stumvoll M,Blüher M. Insulinsensitive obesity. Am J Physiol Endocrinol Metab. 2010;299(3):E506-15.

9. Nishimura S, Manabe I, Nagasaki M, Eto K, Yamashita H, Ohsugi M, Otsu M, Hara K, Ueki K, Sugiura S, Yoshimura K, Kadowaki T, Nagai R. CD8+ effector T cells contribute to macrophage recruitment and adipose tissue inflammation in obesity. Nat Med. 2009; 15(8):914-20.

10. Winer S, Chan Y, Paltser G, Truong D, Tsui H, Bahrami J, Dorfman R, Wang Y, Zielenski J, Mastronardi F, Maezawa Y, Drucker DJ, Engleman E, Winer D, Dosch HM. Normalization of obesity-associated insulin resistance through immunotherapy. Nat Med. 2009; 15(8):921-29.

11. Elgazar-Carmon V, Rudich A, Hadad N, Levy R. Neutrophils transiently infiltrate intra-abdominal fat early in the course of high-fat feeding. J Lipid Res. 2008; 49(9):1894-903.

12. Yamashita A, Soga Y, Iwamoto Y, Asano T, Li Y, Abiko Y, Nishimura F. DNA microarray analyses of genes expressed differentially in 3T3-L1 adipocytes co-cultured with murine macrophage cell line RAW264.7 in the presence of the toll-like receptor 4 ligand bacterial endotoxin. Int J Obes (Lond) 2008; 32(11):1725-29.

13. Suganami T, Nishida J, Ogawa Y. A paracrine loop between adipocytes and macrophages aggravates inflammatory changes: role of free fatty acids and tumor necrosis factor alpha. Arterioscler Thromb Vasc Biol. 2005; 25(10):2062-68.

14. Lumeng CN, Deyoung SM, Saltiel AR. Macrophages block insulin action in adipocytes by altering expression of signaling and glucose transport proteins. Am J Physiol Endocrinol Metab. 2007; 292(1):E166-E174.

15. Lacasa D, Taleb S, Keophiphath M, Miranville A, Clement K. Macrophage-secreted factors impair human adipogenesis: involvement of proinflammatory state in preadipocytes. Endocrinology 2007; 148(2):868-77.

16. Kim JY, van de Wall E, Laplante M, Azzara A, Trujillo ME, Hofmann SM, Schraw T, Durand
JL, Li H, Li G, Jelicks LA, Mehler MF, Hui DY, Deshaies Y, Shulman GI, Schwartz GJ, Scherer PE. Obesity-associated improvements in metabolic profile through expansion of adipose tissue. J Clin Invest. 2007; 117(9):2621- 37.

17. Visser M, Bouter LM, McQuillan GM, Wener $\mathrm{MH}$, Harris TB. Elevated C-reactive protein levels in overweight and obese adults. JAMA 1999; 282(22):2131-35.

18. Pearson TA, Mensah GA, Alexander RW, Anderson JL, Cannon RO 3rd, Criqui M, Fadl YY, Fortmann SP, Hong Y, Myers GL, Rifai N, Smith SC Jr, Taubert K, Tracy RP, Vinicor F; Centers for Disease Control and Prevention; American Heart Association. Markers of inflammation and cardiovascular disease: application to clinical and public health practice: A statement for healthcare professionals from the Centers for Disease Control and Prevention and the American Heart Association. Circulation 2003; 107(3):499-511.

19. Danesh J, Whincup P, Walker M, Lennon L, Thomson A, Appleby P, Gallimore JR, Pepys MB. Low grade inflammation and coronary heart disease: prospective study and updated meta-analyses. BMJ 2000; 321(7255):199-204.

20. Nijhuis J, Rensen SS, Slaats Y, van Dielen FM, Buurman WA, Greve JW. Neutrophil activation in morbid obesity, chronic activation of acute inflammation. Obesity (Silver Spring) 2009; 17(11):201418.

21. Ghanim H, Aljada A, Hofmeyer D, Syed T, Mohanty P, Dandona P. Circulating mononuclear cells in the obese are in a proinflammatory state. Circulation 2004; 110(12):1564-71.

22. Devaraj S, Dasu MR, Rockwood J, Winter W, Griffen SC, Jialal I. Increased toll-like receptor (TLR) 2 and TLR4 expression in monocytes from patients with type 1 diabetes: further evidence of a proinflammatory state. J Clin Endocrinol Metab. 2008; 93(2):578-83.

23. Patel C, Ghanim H, Ravishankar S, Sia CL, Viswanathan P, Mohanty P, Dandona P. Prolonged reactive oxygen species generation and nuclear factor-kappa $B$ activation after a high-fat, high-carbohydrate meal in the obese. J Clin Endocrinol Metab 2007; 92(11):4476-9.

24. Dickinson S, Hancock DP, Petocz P, Ceriello A, Brand-Miller J. High-glycemic index carbohydrate increases nuclear factor-kappaB activation in mononuclear cells of young, lean healthy subjects. Am J Clin Nutr. 2008; 87(5):1188-93.

25. Reyes M, Subramanian V, Rojas C, Ferrante A. 
High fat diet induces early monocytosis. IX Latin American Congress of Immunology.,Viña del Mar (Chile). Libro de resúmenes, p. 160. 2009.

26. Combadière C, Potteaux S, Rodero M, Simon T, Pezard A, Esposito B, Merval R, Proudfoot A, Tedgui A, Mallat Z. Combined inhibition of CCL2, CX3CR1, and CCR5 abrogates Ly6C(hi) and Ly6C(lo) monocytosis and almost abolishes atherosclerosis in hypercholesterolemic mice. Circulation 2008; 117(13):1649-57.

27. Libby P. Inflammation in atherosclerosis. Nature. 2002; 420(6917):868-74.

28. Devaraj S, Cheung AT, Jialal I, Griffen SC, Nguyen D, Glaser N, Aoki T. Evidence of increased inflammation and microcirculatory abnormalities in patients with type 1 diabetes and their role in microvascular complications. Diabetes 2007; 56(11): 2790-96.

29. Lynch LA, O'Connell JM, Kwasnik AK, Cawood TJ, O'Farrelly C, O'Shea DB. Are natural killer cells protecting the metabolically healthy obese patient? Obesity (Silver Spring). 2009; 17(3):601-5.

30. Fernández-Real JM, Pickup JC. Innate immunity, insulin resistance and type 2 diabetes. Trends Endocrinol Metab 2008; 19(1):10-6.

31. Esteve E, Ricart W, Fernández-Real JM. Dyslipidemia and inflammation: an evolutionary conserved mechanism. Clin Nutr. 2005; 24(1):16-31.

32. Zhang C. The role of inflammatory cytokines in endothelial dysfunction. Basic Res Cardiol. 2008; 103(5):398-406.

33. Baffy G. Kupffer cells in non-alcoholic fatty liver disease: the emerging view. J Hepatol. 2009; 51(1):212-23.

34. Hotamisligil GS. Inflammation and metabolic disorders. Nature. 2006; 444(7121):860-67.

35. Keusch GT. The history of nutrition: malnutrition, infection and immunity. J Nutr. 2003; 133(1):336S$340 \mathrm{~S}$.

36. Lacy-Hulbert A, Moore KJ. Designer macrophages: oxidative metabolism fuels inflammation repair. Cell Metab. 2006; 4(1):7-8.

37. Wildman RP, Muntner P, Reynolds K, McGinn AP, Rajpathak S, Wylie-Rosett J, Sowers MR. The obese without cardiometabolic risk factor clustering and the normal weight with cardiometabolic risk factor clustering: prevalence and correlates of 2 phenotypes among the US population (NHANES 19992004). Arch Intern Med. 2008; 168(15):1617-24.

38. Goyenechea E, Parra D, Crujeiras AB, Abete I, Martínez JA. A nutrigenomic inflammation-related PBMC-based approach to predict the weight-loss regain in obese subjects. Ann Nutr Metab. 2009; 54(1):43-51. 